\title{
Real-time Thermographic Methodology with High-accuracy Temperature Monitoring Applied to Stacked Package of High-power Semiconductor Laser Diode
}

\author{
Shun-Lung Yen, ${ }^{1}$ Shiang-Feng Tang, ${ }^{2 *}$ Chung-Wei Ou, ${ }^{3}$ Chin-Jung Chao, ${ }^{3}$ \\ Hsin-Yen Cheng, ${ }^{2}$ Ing-Jiunn Su, ${ }^{1}$ and Tzu-Chiang Chen ${ }^{1}$ \\ ${ }^{1}$ Department of Electrical and Electronic Engineering, Chung Cheng Institute of Technology, \\ National Defense University, Tauyuan County 33000, Taiwan, R.O.C. \\ ${ }^{2}$ Materials \& Electro-Optics Research Division, National Chung Shun Institute of Technology, \\ Tauyuan County 32599, Taiwan, R.O.C. \\ ${ }^{3}$ Department of Industrial and Systems Engineering, Chung Yuan Christian University, \\ Tauyuan County 32023, Taiwan, R.O.C.
}

(Received July 10, 2018; accepted March 7, 2019)

Keywords: high-power semiconductor laser diode (HPSLD), regression coefficients, uncooled thermal camera, focal plane array (FPA), readout integrated circuit (ROIC), microchannel cooling (MCC)

To improve the accuracy of temperature measurement using a commercial thermal imager, the proposed correction methodology for the regression coefficients $R, B, F$, and $O$ is applied, and the accuracy reaches $< \pm 1{ }^{\circ} \mathrm{C}$ in the temperature measurement range between 15 and $100{ }^{\circ} \mathrm{C}$. The use of digital images with a Giga-Ethernet output interface for the development of temperature measurement and analysis software is the strategy of real-time temperature measurement and fast monitoring of the temperature of the target area. It will become a costeffective room-temperature thermal imager with high accuracy of temperature measurement suitable for microzone application. The use of a thermal camera for the temperature observation of a microzone on a high-power striped laser diode (LD) is advantageous in the evaluation of the heat dissipation of a submount stage to improve the output efficiency of the laser.

\section{Introduction}

Over the last two decades, an uncooled thermal camera has been widely used in the industrial, medical, and defense fields. ${ }^{(1)}$ Generally, it is mainly applied to long-range target tracking, surveillance, and temperature measurement. Previously, the adoption of a highend uncooled thermal camera was limited owing to its expensive micro-infrared optics, the requirement of high spatial pixel resolution, and issues concerning optic depth resulting in the need for precise $Z$-axis positioning. In particular, for the measurement of temperature on a microzone of $50 \mu \mathrm{m}$ scale or smaller, a thermal camera must be used with phase-locked microscopy and synchronized thermography. Therefore, this system has been very costly.

*Corresponding author: e-mail: shiangfengtang@gmail.com https://doi.org/10.18494/SAM.2019.2055 
In this study, we used a commercial room-temperature-operated long-wavelength infrared imaging module incorporating a lens with a focal length of $19 \mathrm{~mm}$ and a lens sleeve designed to reduce thermal stray radiation, to shorten the distance between the lens and the object measured (from 50 to $2.85 \mathrm{~cm}$ ). Hence, the spatial resolution approaches $8.5 \mu \mathrm{m}$, providing a zoom-in function to observe the temperature of a microzone containing the interfaces of a submount solder for a laser diode (LD). With the monitoring of the temperature of its housing and the operating temperature of the internal focal plane array (FPA) in the thermal imaging module under a controllable ambient temperature, the imaging module can be used with the linear regression approach for thermal radiation correction at different steady-state ambient temperatures. For example, in the microzone temperature measurement, the highpower semiconductor LD is regarded as the exciting laser trigger source. Also, the LD is the critical chip. Because of heat dissipation due to defects of the high-power LD, excess heat at the interface of the attached region cannot be removed efficiently, i.e., laser output would be decreased. In this study, a microzone temperature-monitoring platform with a real-time temperature calibration algorithm was proposed and applied to the package on the submount stage of a high-power semiconductor laser diode (HPSLD) to evaluate its power efficiency. ${ }^{(2,3)}$ Because the size of the temperature monitoring scale is only $100 \mu \mathrm{m}-2 \mathrm{~mm}$, it is suitable for the above needs, that is, it is not necessary to use an expensive lock-in thermographic camera.

\section{Experimental Setup and Temperature Monitoring Methodology}

Using a commercial long-wavelength infrared thermal camera with an IR optic lens having an object distance from $50 \mathrm{~cm}$ to $\infty$, we design an optically stray-radiation-suppressing sleeve to shorten the distance from the FPA to the optic lens, for microzone imaging and accurate temperature measurement. The temperature accuracy approaches a value less than $\pm 1{ }^{\circ} \mathrm{C}$. The thermal camera with the optically stray-radiation-suppressing sleeve is shown in Fig. 1.

According to Planck's Radiation Law, the radiant exitance or emissivity of an object is related to the surface of the material itself. Using Planck's spectral distribution, a thermal signal is converted to an electrical signal by a thermal detector to generate a thermal distribution

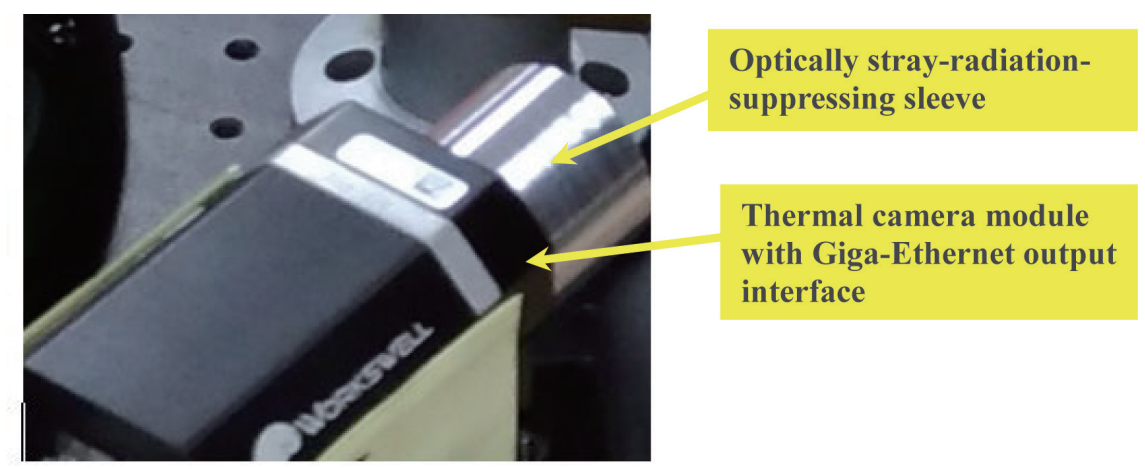

Fig. 1. (Color online) Thermal camera with optically stray-radiation-suppressing sleeve. 
pattern visible to humans. For the proposed temperature detection technology, the FPA based on a microbolometer is used to measure the thermal absorption that causes a change in resistance, and then a voltage or current (numerical intensity) signal is obtained. The voltage or current signal from the microbolometer is subsequently converted using the numerical fitting equation of Planck's distributed curve. The reverse substitution is conducted to obtain the actual temperature $\left(T_{0}\right)$ as ${ }^{(4)}$

$$
V_{D}=\frac{R}{e^{\frac{B}{T_{0}}}-F}+O,
$$

where $R, B, F$, and $O$ are the main thermal radiation regression coefficients. The actual temperature is obtained as

$$
T_{0}=\frac{B}{\ln \left(\frac{R}{V_{D}-O}+F\right)},
$$

where $O$ indicates the offset value of numerical intensity, which leads to the parallel translation along the $y$-axis of the numerical fitting equation of Planck's distributed curve; $R$ is the response of the thermal camera, which is related to the system sensitivity $R_{S} ; B$ is related to the effective wavelength sensitivity of the thermal camera; $F$ is the nonlinear calibration factor, which is generally set to 1 .

\section{Temperature Calibration of Thermal Camera Module}

Because of the difference in the processes for the microbolometer and FPA, which are affected by the operating temperature, the signal output from a readout integrated circuit (ROIC) contains some numerical error that results in an inaccurate temperature measurement. Thus, using a proper calibration methodology, the accuracy of temperature measurement must be improved. In other words, ambient, shutter surface, and indoor FPA temperatures are monitored to determine the thermal radiation regression coefficients $R, B, F$, and $O .^{(5,6)}$ The thermal camera module based on the microbolometer is placed in a temperature-controllable box (the temperature ranges from 0 and $50{ }^{\circ} \mathrm{C}$ ). The two flat plated blackbodies are put in front of the thermal camera module. The radiant exitances from the two flat plated blackbodies tuned at two different temperatures for the derivation of $R$ and $O$ in Eq. (2) of the thermal camera module in the temperature-controllable box are determined for the accurate calibration of temperature. ${ }^{(7)}$ The following are the calibration steps.

1. Two blackbodies of low and high temperature ranges $\left(0-50\right.$ and $\left.50-500{ }^{\circ} \mathrm{C}\right)$ are used for high- and low-temperature-point calibrations.

2. The temperature-controllable box is set up with temperatures in the range of $15-45{ }^{\circ} \mathrm{C}$ at intervals of $5^{\circ} \mathrm{C}$. 
3. At each testing temperature interval, the overall ambient temperature is in the steady state after the target temperature is held for $20 \mathrm{~min}$. Then, the nonuniformity is processed.

4. At the same time, the temperatures at the surface of the optic shutter and the indoor FPA are recorded.

5. For the low and high temperature ranges, 30 and $90{ }^{\circ} \mathrm{C}$ blackbodies are set up and $V_{D}$ is recorded automatically over 2 min (120 data sets are obtained). The obtained data are averaged and shown in Fig. 2. Although the average counts show some deviation, the curves are generally monotonic.

The average $V_{D}$ values for the 30 and $90{ }^{\circ} \mathrm{C}$ blackbody temperatures in Fig. 2 are substituted into Eq. (1), with constant values $B$ and $F$ (experimentally, numerical values are related to the uncooled camera) of 1428 and 1, respectively. Two identical equations are solved to obtain $R$ and $O$ under seven different ambient temperatures. Numerical approximations of $R$ and $O$ are followed by linear regression analysis under the steady-state ambient temperatures of $15-45^{\circ} \mathrm{C}$, as shown in Eqs. (3) and (4).

$$
\begin{gathered}
R=338722.3+(-1237.4) \times T \\
O=346.303+21.476 \times T
\end{gathered}
$$

In Figs. 3 and 4, $R_{S}$ ( $R$-square, coefficient of determination) and standard deviation (SD) are respectively 0.99888 and 695.0405 for $R$ and 0.99469 and 26.29014 for $O$, which means that the values are accurately estimated for any steady-state ambient temperature in the range of $15-45^{\circ} \mathrm{C}$. Sequentially, the temperature-controllable box is set up for $17,22,27,32,37$, and $42{ }^{\circ} \mathrm{C}$ between 15 and $45^{\circ} \mathrm{C}$, and held in the steady state for $20 \mathrm{~min}$. The temperatures of the low-temperature blackbody are 20 and $50{ }^{\circ} \mathrm{C}$, and those of the high-temperature blackbody are 70 and $100{ }^{\circ} \mathrm{C}$. As described above, the 120 data sets of $V_{D}$ (in counts) are recorded in 2 min for these blackbody

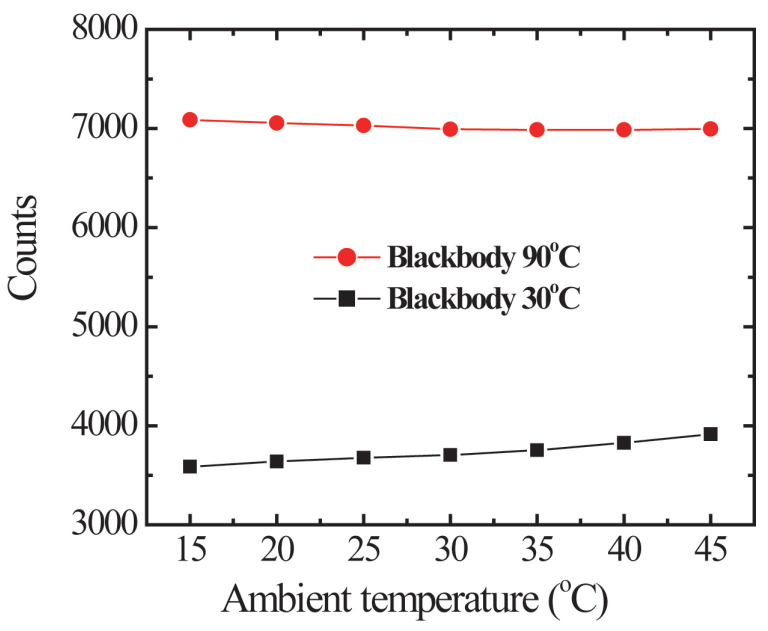

Fig. 2. (Color online) Average $V_{D}$ values for various counts of thermal camera for 30 and $90{ }^{\circ} \mathrm{C}$ blackbody temperatures under different ambient temperatures. 


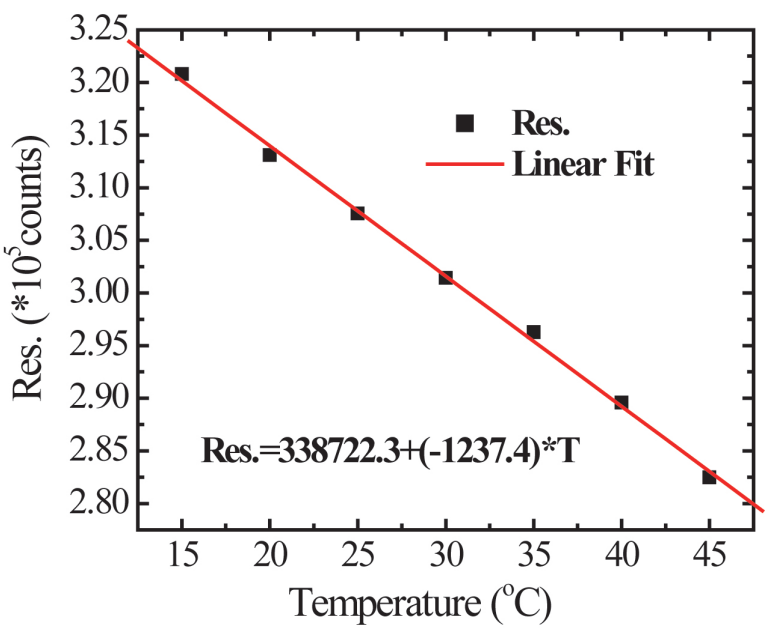

Fig. 3. (Color online) Numerical approximation of $R \equiv$ Res. using the optimized $R_{S}$ (i.e., goodness of fit of coefficient of determination) by linear regression analysis at the steady-state ambient temperatures of $15-45^{\circ} \mathrm{C}$.

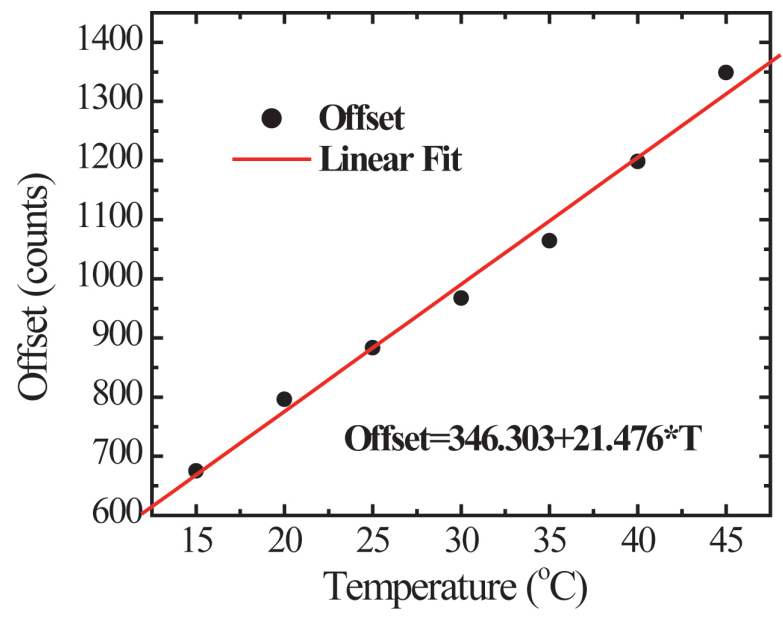

Fig. 4. (Color online) Numerical approximation of $O$ using the optimized $R_{S}$ (i.e., goodness of fit of coefficient of determination) by linear regression analysis at the steady-state ambient temperatures of $15-45^{\circ} \mathrm{C}$.

temperatures. Using Eqs. (3) and (4), we can calculate the relevant $R$ and $O$ values under six different ambient temperatures where $B$ and $F$ have empirical values of 1428 and 1 . From the inverse operation of Eq. (1), the actual temperatures including max., min., ave., and blackbody temperatures are obtained with the known relevant $R$ and $O$ values shown in Figs. 5-8. The average errors are $0.31,0.61,0.11$, and $0.11{ }^{\circ} \mathrm{C}$ when the blackbody temperatures are $20,50,70$, and $100{ }^{\circ} \mathrm{C}$, and the maximal errors are $0.78,1.0,0.36$, and $0.44{ }^{\circ} \mathrm{C}$, respectively. It is obvious that the accuracy of temperature measurement is very high and may be better than $\pm 1{ }^{\circ} \mathrm{C}$. It is also interesting to note that the maximal errors are larger for lower blackbody temperatures than for higher blackbody temperatures. This result could be explained by the measurement of lower target temperatures (in this case, blackbody temperatures of 20 and $50{ }^{\circ} \mathrm{C}$ ) being more easily disturbed by the ambient temperatures of $15-45^{\circ} \mathrm{C}$.

\section{Temperature Monitoring of Microzone between Multimetal Solders on Top of Laser-diode Bar and Microchannel Cooling (MCC) Stage}

Figure 9 shows the experimental setup for monitoring the temperatures of multimetal solders of the LD bar and MCC stage. The thermal camera module with the above temperaturecalibration methodology is used and mounted on the triaxial optical positioning platform. Owing to the very high power coherent exitance of the LD bar upon serious damage of the microbolometer FPA behind the IR lens, the optic path is off-axis by $30^{\circ}$, which includes the normal direction of the focal plane of the thermal camera. The optical imaging distance between the objective and the IR lens is about $2.85 \mathrm{~cm}$. The digital imaging data and spatial 


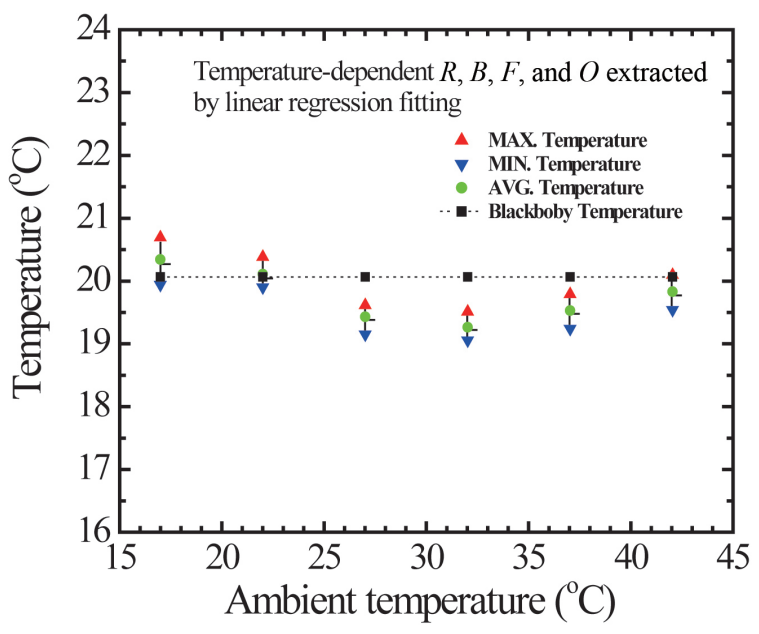

Fig. 5. (Color online) Maximal, minimal, and average temperatures calibrated using 120 data sets recorded with temperature-dependent $R, B, F$, and $O$ at different ambient temperatures. The target (blackbody) temperature was $20^{\circ} \mathrm{C}$.

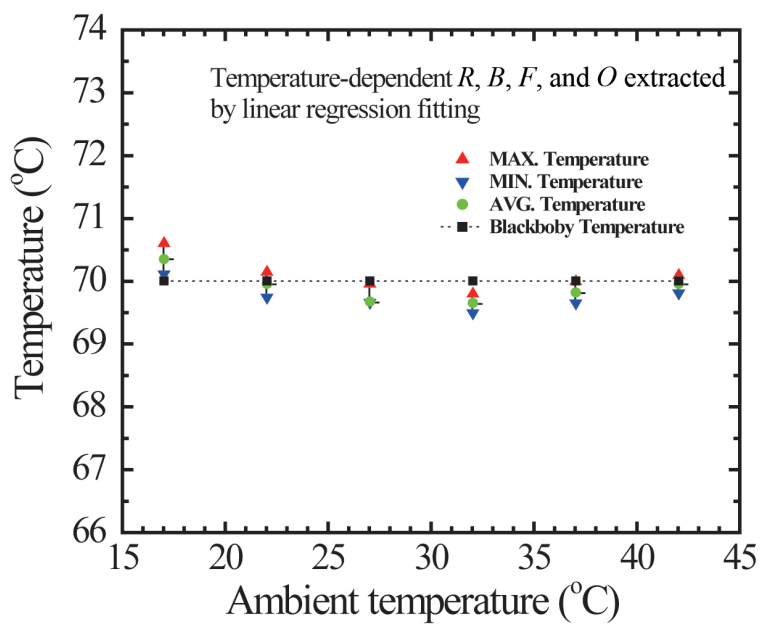

Fig. 7. (Color online) Maximal, minimal, and average temperatures calibrated using 120 data sets recorded with temperature-dependent $R, B, F$, and $O$ at different ambient temperatures. The target (blackbody) temperature was $70^{\circ} \mathrm{C}$.

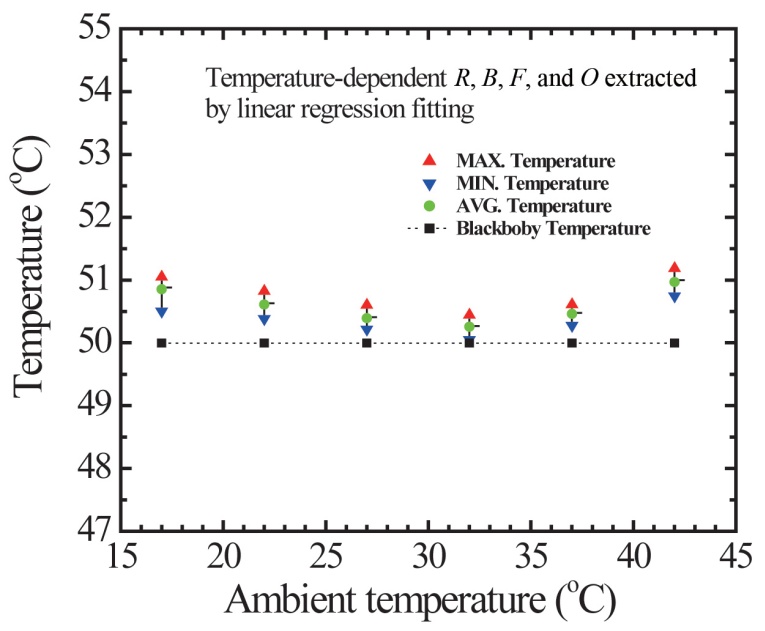

Fig. 6. (Color online) Maximal, minimal, and average temperatures calibrated using 120 data sets recorded with temperature-dependent $R, B, F$, and $O$ at different ambient temperatures. The target (blackbody) temperature was $50^{\circ} \mathrm{C}$.

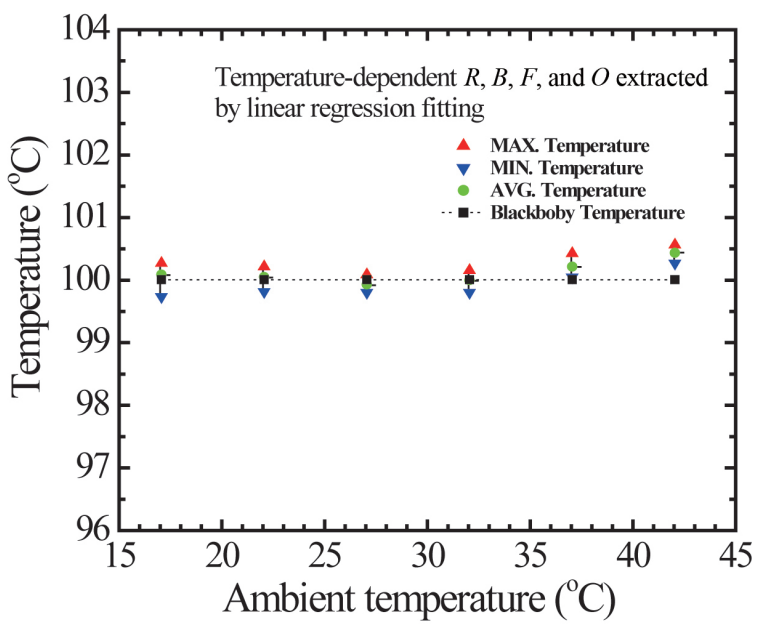

Fig. 8. (Color online) Maximal, minimal, and average temperatures calibrated using 120 data sets recorded with temperature-dependent $R, B, F$, and $O$ at different ambient temperatures. The target (blackbody) temperature was $100{ }^{\circ} \mathrm{C}$.

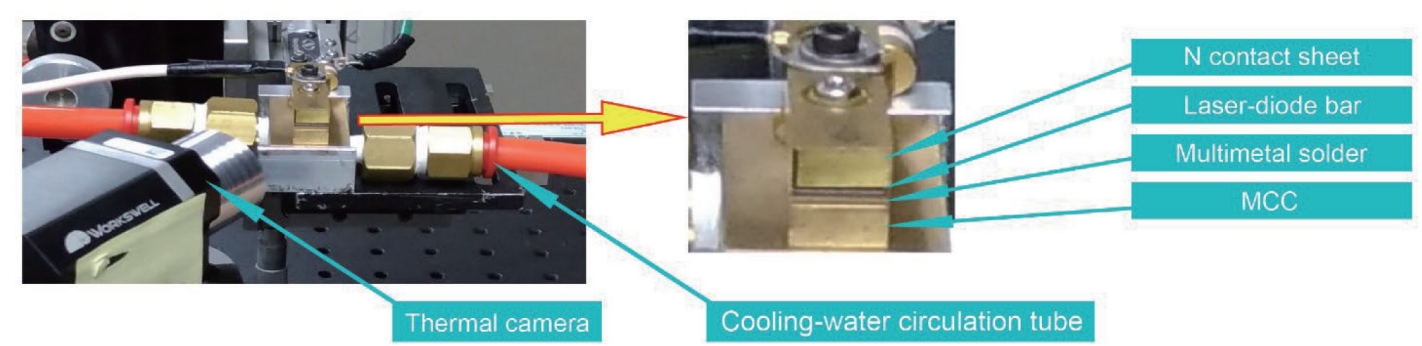

Fig. 9. (Color online) System structure setup of LD bar with multimetal solder, MCC, cooling-water circulation tube, and microzone functional thermal camera. 
intensity are transferred into NI-PXI through the RJ-45 port of Giga-Ethernet for microzone functional thermal imaging and real-time calibration associated with high-precision temperature measurement. Figure 10 shows the thermal image of the high-power LD bar corresponding to the schematic diagram of the Type 1 package. There are two positions where the temperatures are monitored: the beam surface of the LD marked by "+" and the junction below the hard solder, i.e., the topmost surface of joint multimetal layers, marked by " $\times$ ". In this research, we use two kinds of joint mounted structure for the package of the high-power LD with the peak wavelength of $808 \mathrm{~nm}$. One comprises $\mathrm{Cu} / \mathrm{AlN} / \mathrm{Cu}$ multimetals soldered with $\mathrm{Au}_{80} \mathrm{Sn}_{20}$, as shown in Fig. 10; the other comprises submounted $\mathrm{Cu}_{10} \mathrm{~W}_{90}$ sandwiched with two 5- $\mu \mathrm{m}$-thick $\mathrm{Au}_{80} \mathrm{Sn}_{20}$ solders defined with the Type 2 package, as shown in Fig. 11.

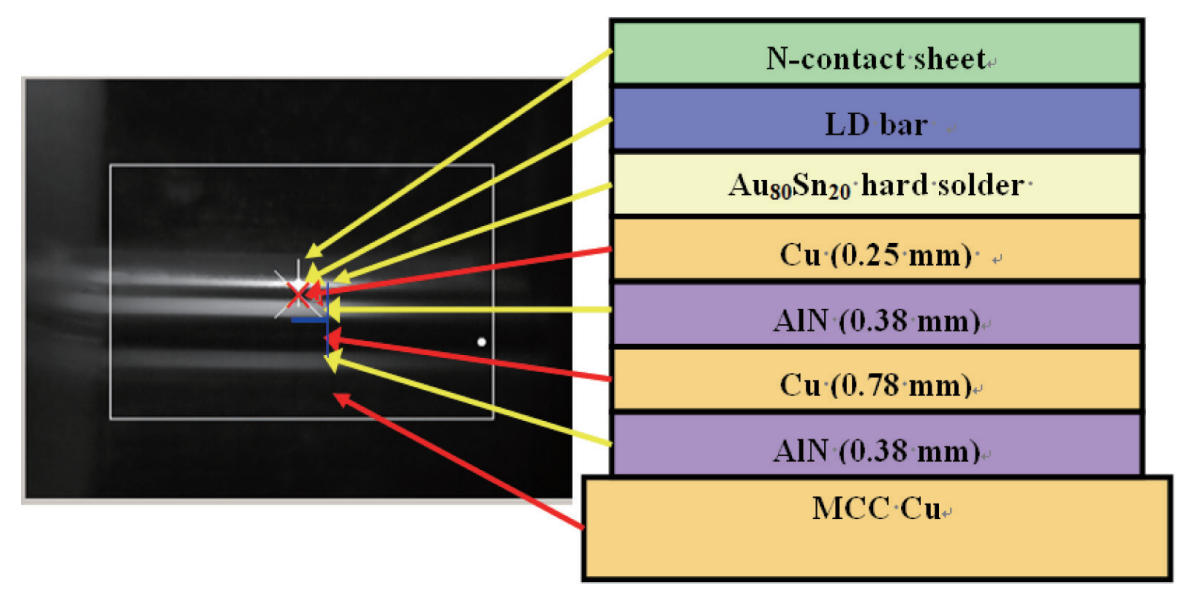

Fig. 10. (Color online) Thermal image and schematic diagram of high-power LD bar comprising $\mathrm{Cu} / \mathrm{AlN} / \mathrm{Cu}$ multimetals soldered with $\mathrm{Au}_{80} \mathrm{Sn}_{20}$ (Type 1 package).

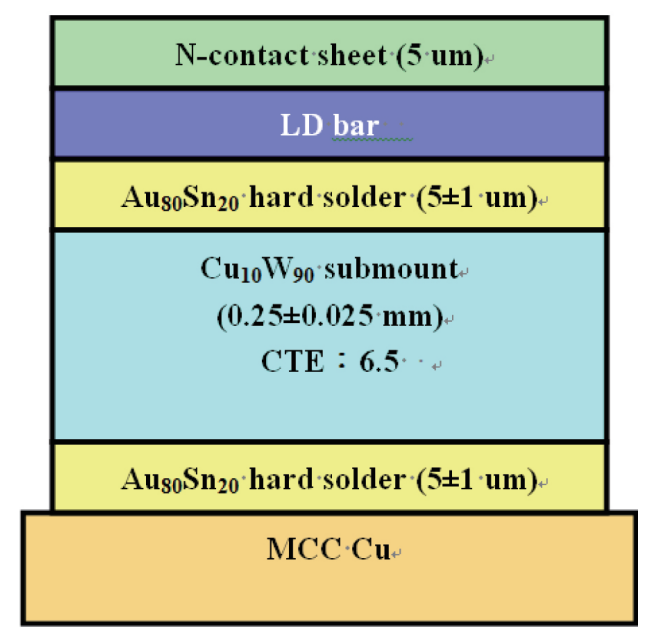

Fig. 11. (Color online) Schematic diagram of Type 2 package with the high-power LD bar using submounted $\mathrm{Cu}_{10} \mathrm{~W}_{90}$ sandwiched with two 5- $\mu$ m-thick $\mathrm{Au}_{80} \mathrm{Sn}_{20}$ solders. 


\section{Real-time Temperature Analyses on Two Types of Submounted LD Bar}

For Type 1 package, the temperature of the beam surface on the edge LD bar approaches $43{ }^{\circ} \mathrm{C}$ as the driving DC current increases to $34 \mathrm{~A}$. In contrast, the temperature of the $\mathrm{Cu} /$ AlN/Cu metal joint is only $24.5{ }^{\circ} \mathrm{C}$, which is close to the ambient temperature of $22.5{ }^{\circ} \mathrm{C}$, as shown in Fig. 12. The higher DC driving current indicates a larger temperature difference between the beam surface on the edge LD bar and the $\mathrm{Cu} / \mathrm{AlN} / \mathrm{Cu}$ metal joint. This means that once the lasing process begins, the heat absorbed by the LD bar is not conducted effectively into the $\mathrm{Cu} / \mathrm{AlN} / \mathrm{Cu}$ metal joint mounted in the water-cooled MCC module, resulting in the decreasing efficiency of LD power. However, for the Type 2 package shown in Fig. 13, the temperature of the beam surface on the edge LD bar remains at only $47.7{ }^{\circ} \mathrm{C}$ as the driving DC current increases beyond $90 \mathrm{~A}$. The temperature of the joint on the sandwiched $\mathrm{Cu}_{10} \mathrm{~W}_{90}$ approaches $44.5^{\circ} \mathrm{C}$, which is higher than the ambient temperature of $23.0^{\circ} \mathrm{C}$. These differences in temperature between the beam surface of the $\mathrm{LD}$ and the joint on the sandwiched $\mathrm{Cu}_{10} \mathrm{~W}_{90}$ are almost the same. This can be explained by the submount package providing the better heatconducting junction for the removal of heat generated from the high-power LD bar. The peak wavelength suddenly shifts from 803.25 to $804.75 \mathrm{~nm}$ when the DC driving current exceeds 55 A. Furthermore, a driving current higher than 85 A causes the peak wavelength to shift to $805.45 \mathrm{~nm}$. It is concluded that the hop mode appears in the single-mode LD owing to the changes in operation temperature. A high power of $86.6 \mathrm{~W}$ and a peak wavelength of 805.25 $\mathrm{nm}$ can be obtained under the DC driving current of $90 \mathrm{~A}$. By real-time microzone temperature monitoring, the changes in the temperatures of the beam surface of the high-power LD bar and its joint submount are resolved well. The temperature-driving current curve provides the optimal choice of metal joint materials and the process of Type 2 package on the junction of the high-power LD bar.

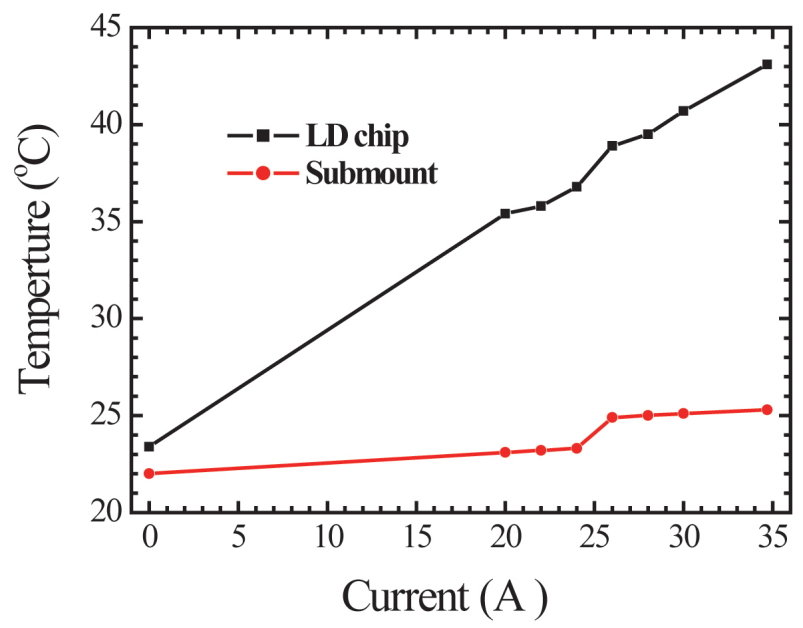

Fig. 12. (Color online) Temperature vs DC driving current curves of beam surface on the edge LD bar as the driving DC current increases to $34 \mathrm{~A}$. In contrast, the temperature of the $\mathrm{Cu} / \mathrm{AlN} / \mathrm{Cu}$ metal joint is only $24.5{ }^{\circ} \mathrm{C}$, which is close to the ambient temperature of $22.5^{\circ} \mathrm{C}$. 


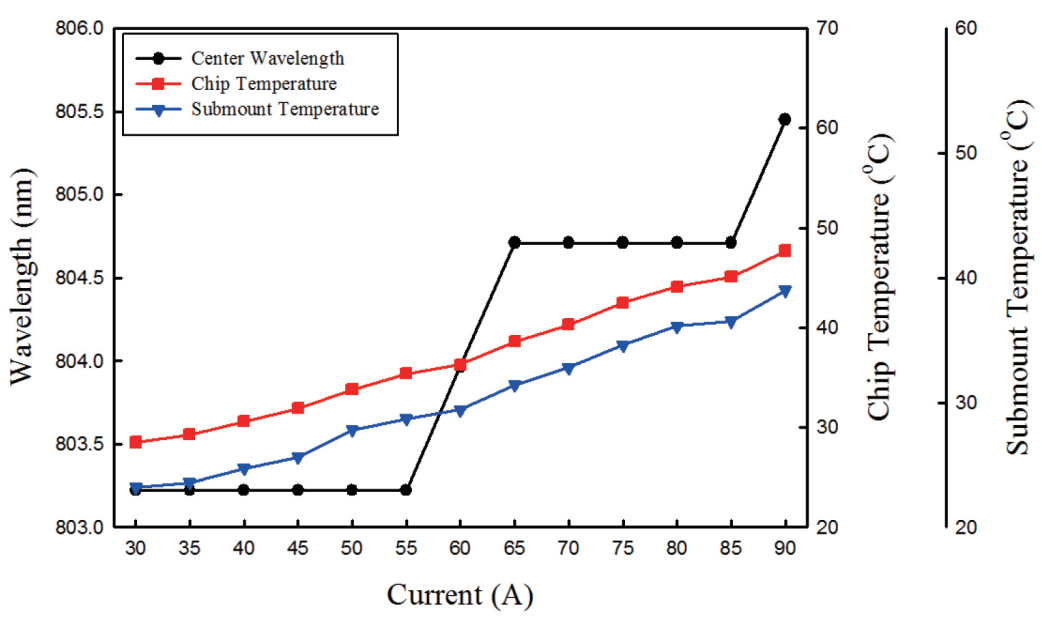

Fig. 13. (Color online) Temperature vs DC driving current and peak-wavelength curves of beam surface on the edge $\mathrm{LD}$ bar and sandwich $\mathrm{Cu}_{10} \mathrm{~W}_{90}$ submounted package.

\section{Conclusion}

An optical zoom-in and stray-radiation sleeve was slipped onto an IR lens with a constant focal length, and the proposed correction methodology for the regression coefficients $R, B$, $F$, and $O$ of a microzone temperature-monitoring thermal camera was used for real-time temperature measurement. In our research, we have used a commercial room-temperatureoperated long-wavelength infrared imaging module incorporating a lens with a focal length of $19 \mathrm{~mm}$ and a lens sleeve designed to reduce thermal stray radiation, to shorten the distance between the lens and the object measured (from 50 to $2.85 \mathrm{~cm}$ ). Hence, the spatial resolution approaches $8.5 \mu \mathrm{m}$, providing a zoom-in function to observe the temperature of a microzone containing the interfaces of a submount solder for an LD. The accuracy of temperature measurement is higher than $\pm 1{ }^{\circ} \mathrm{C}$. Applying the proposed microzone temperature-monitoring thermal camera for the evaluation of the package of an HPLD, we clearly observed the real-time temperatures of the junction of multimetal joints and the beam surface of the LD. In the future, by realizing heat distribution and thermal crowding on the junctions of the submount and LD to optimize the joint materials and packaging process, a high-performance package for the highpower LD bar or array will be obtained.

\section{Acknowledgments}

This research is partially supported by National Chung Shan Institute of Science and Technology, sponsored by the Ministry of Economic Affairs, Taiwan, R.O.C., under Grant no. NCSIST-310-V302(107). 


\section{References}

1 A. Rogalski: Infrared Phys. Technol. 43 (2002) 187.

2 J. Morales, S. Lehkonen, G. Liu, D. Schleuning, and B. Acklin: Proc. SPIE 9733, High-Power Diode Laser Technology and Applications XIV (SPIE, 2016) 97330T.

3 D. Hou, J. Wang, L. Gao, X. Liang, X. Li, and X. Liu: Proc. SPIE 9730, Components and Packaging for Laser Systems II (SPIE, 2015) 973009.

4 H. Budzier and G. Gerlach: AMA Conf. 2015 Proc. IRS² (AMA, 2015) 889.

5 H. Budzier and G. Gerlach: J. Sens. Sens. Syst. 4 (2015) 187.

6 A. Tempelhahn, H. Budzier, V. Krause, and G. Gerlach: J. Sens. Sens. Syst. 5 (2016) 9.

7 D. Lin, P. Westfeld, and H.-G. Maas: Int. Arch. Photogramm. Remote Sens. Spatial Inf. Sci., XLII-1/W1 (2017) 619.

\section{About the Authors}

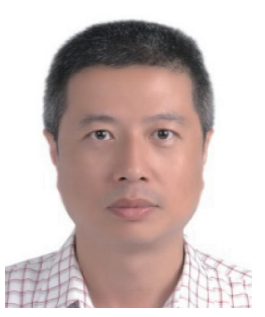

Shun-Lung Yen is a doctoral graduate student in the Department of Electrical and Electronic Engineering, Chung Cheng Institute of Technology of National Defense University. His research interests are in the correction of temperature measurement using an infrared thermal camera and its application in the industrial field.

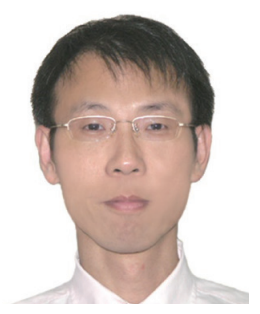

Shiang-Feng Tang received his B.S. degree in electrical and electronic engineering from Chung Cheng Institute of Technology, Taiwan, in 1991 and his M.S. and Ph.D. degrees from the National Taiwan University, Taiwan, in 1996 and 2001, respectively. Since 2002, he has been an assistant researcher at National Chung Shan Institute of Science and Technology, Taiwan. Since 2010, he has also been an adjunct associate professor in the Department of Electrical and Electronic Engineering, Chung Cheng Institute of Technology of National Defense University. His research interests are in MEMS processing, nano-optoelectronic devices, and infrared sensors.

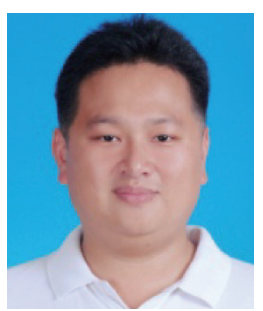

Chung-wei Ou is currently studying at Chung Yuan Christian University Department of Industrial and Systems Engineering, as a doctoral candidate. He is now an Army Colonel in the Ministry of Defense and works part-time in the National Defense Industrial Development Foundation. His research interests include anthropometry, risk management, service quality, and ergonomics. 


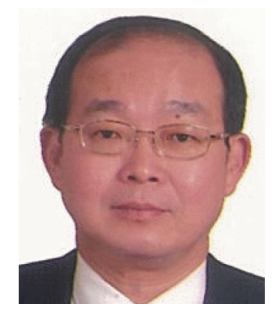

Chin-jung Chao received his Ph.D. degree in industrial engineering from Purdue University. He is now an associate professor in the Department of Industrial and Systems Engineering, Chung Yuan Christian University.

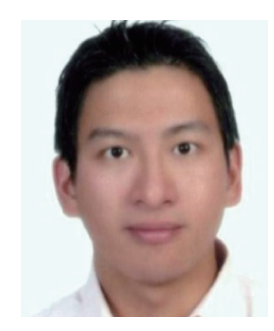

Hsin-Yen Cheng received his B.S. and M.S. degrees in material engineering from I-SHOU University, Kaohsiung, in 2003 and 2005, respectively, and his Ph.D. degree from the Department of Materials Science and Engineering, in NCKU, Tainan, in 2017. His research interests are in high-power diode laser stack packaging, solar absorbers, sputter thin-film process, and nanomaterials/ nanostructures.

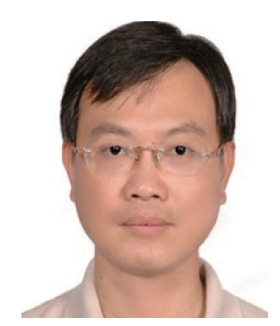

Ing-Jiunn Su received his B.S. degree in communication engineering from National Chiao Tung University, Hsinchu, and his M.S. and Ph.D. degrees in electrical engineering from National Taiwan University, Taipei, Taiwan, in 1985, 1987, and 1998, respectively. He joined the Industrial Technology Research Institute in 1989, where he worked on communication networks. Currently, he is an associate professor in the Department of Electrical and Electronic Engineering, Chung Cheng Institute of Technology, National Defense University, Taoyuan, Taiwan. His research interests are in wireless communications, communication signal processing, spread spectrum techniques, and infrared thermometer measurement.

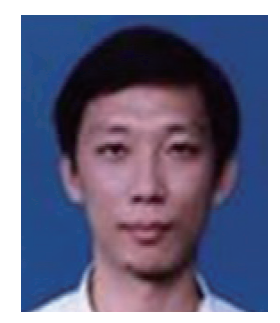

Tzu-Chiang Chen received his B.S. degree from Chung Cheng Institute of Technology, Taoyuan, Taiwan, in 1988, and his M.S. and Ph.D. degrees in space science and optical science from National Central University, Jhongli, Taiwan, in 1992 and 2000, respectively. He became a lecturer in the Department of Applied Physics, Chung Cheng Instituter of Technology in 1992 and an associate professor in 2001. He is currently an associate professor in the Department of Electrical and Electronic Engineering of the National Defense University, Taoyuan, Taiwan. His research interests include optical sensors and the modeling and fabrication of novel quantum optoelectronic devices. 